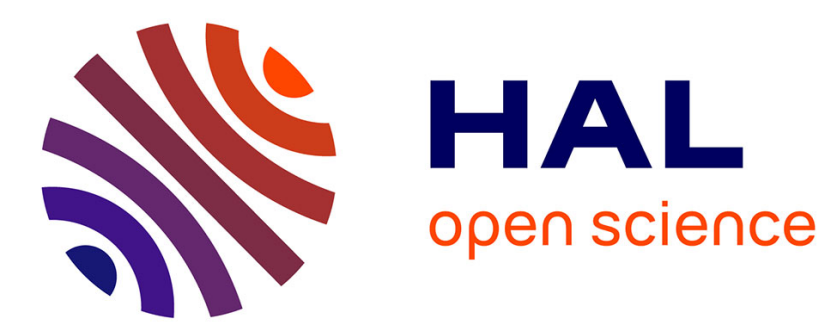

\title{
Flutter of long flexible cylinders in axial flow
}

\author{
Emmanuel de Langre, M.P. Païdoussis, Olivier Doaré, Y. Modarres-Sadeghi
}

\section{To cite this version:}

Emmanuel de Langre, M.P. Païdoussis, Olivier Doaré, Y. Modarres-Sadeghi. Flutter of long flexible cylinders in axial flow. Journal of Fluid Mechanics, 2007, 571 (january), pp.371-389. 10.1017/S002211200600317X . hal-01025717

\section{HAL Id: hal-01025717 \\ https://hal-polytechnique.archives-ouvertes.fr/hal-01025717}

Submitted on 18 Jul 2014

HAL is a multi-disciplinary open access archive for the deposit and dissemination of scientific research documents, whether they are published or not. The documents may come from teaching and research institutions in France or abroad, or from public or private research centers.
L'archive ouverte pluridisciplinaire HAL, est destinée au dépôt et à la diffusion de documents scientifiques de niveau recherche, publiés ou non, émanant des établissements d'enseignement et de recherche français ou étrangers, des laboratoires publics ou privés. 


\title{
Flutter of long flexible cylinders in axial flow
}

\author{
By E. DE LANGRE ${ }^{1,2}$, M. P. PAÏDOUSSIS ${ }^{2}$, \\ O. DOARÉ ${ }^{3}$ AND Y. MODARRES-SADEGHI ${ }^{2}$ \\ ${ }^{1}$ Department of Mechanics, LadHyX, Ecole Polytechnique, Palaiseau 91128, France \\ ${ }^{2}$ Department of Mechanical Engineering, McGill University, Montréal H3A 2K6, Canada \\ ${ }^{3}$ UME, ENSTA, Palaiseau 91761, France
}

(Received 3 October 2005 and in revised form 3 July 2006)

We consider the stability of a thin flexible cylinder considered as a beam, when subjected to axial flow and fixed at the upstream end only. A linear stability analysis of transverse motion aims at determining the risk of flutter as a function of the governing control parameters such as the flow velocity or the length of the cylinder. Stability is analysed applying a finite-difference scheme in space to the equation of motion expressed in the frequency domain. It is found that, contrary to previous predictions based on simplified theories, flutter may exist for very long cylinders, provided that the free downstream end of the cylinder is well-streamlined. More generally, a limit regime is found where the length of the cylinder does not affect the characteristics of the instability, and the deformation is confined to a finite region close to the downstream end. These results are found complementary to solutions derived for shorter cylinders and are confirmed by linear and nonlinear computations using a Galerkin method. A link is established to similar results on long hanging cantilevered systems with internal or external flow. The limit case of vanishing bending stiffness, where the cylinder is modelled as a string, is analysed and related to previous results. Comparison is also made to existing experimental data, and a simple model for the behaviour of long cylinders is proposed.

\section{Introduction}

When a long flexible cylinder with a free downstream end is surrounded by flow parallel to its axis (figure 1) transverse deflection may occur. This may be the result of random excitation forces, such as those caused by turbulence. It may also be the consequence of full coupling between the motion of the cylinder and the unsteady dynamics of the flow. The latter case may lead to what is commonly referred to as a fluidelastic instability. It is of practical importance in the design of systems such as towed underwater acoustic arrays or long floating containers for oil or water transportation. Excessive departure from the expected straight-line configuration results in additional drag, poor performance of the acoustic sensors or early failure through fatigue. Other applications may be found in high-speed train vibrations or wear of flexible rods in nuclear reactors.

A general review of most of the existing work on the subject may be found in Païdoussis (2003), where various geometries and boundary conditions are considered. We focus here on the case of a cantilevered (i.e. clamped-free) straight cylinder. Experimental results in Païdoussis (1966) and Païdoussis et al. (2002) have shown that, as the flow velocity is increased, the system loses stability first by divergence (growing non-oscillatory motion), then by flutter (oscillatory motion). This occurs only when the 


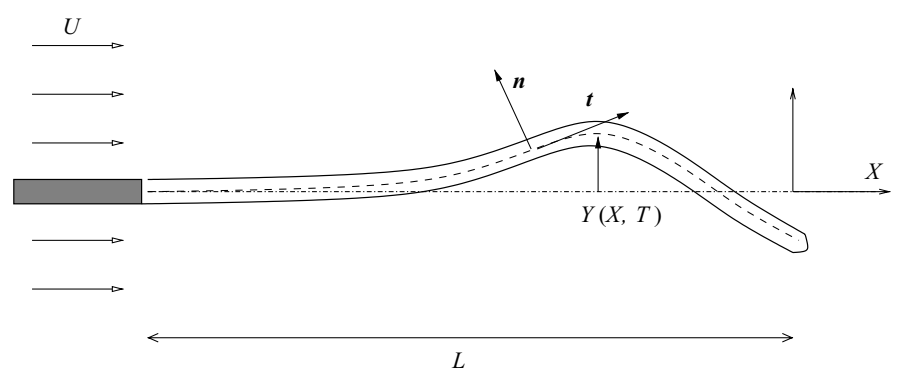

Figure 1. A long cantilevered cylinder in axial flow.

end-shape is sufficiently well streamlined. Other experiments by Ni \& Hansen (1978), Sudarsan, Bhattacharyya \& Vendhan (1997) and Pao (1970) also showed cases of divergence and flutter. In similar but distinct problems, such as that of a filament in a plane flowing soap film (Zhang et al. 2000) or a filament inclined in the flow (Schouveiler, Eloy \& Le Gal 2005), flutter has also been observed. In terms of models, the earlier linear analysis of Païdoussis (1966), corrected in Païdoussis (1973), has been used in various forms by most of the authors: Triantafyllou \& Chryssostomidis (1984, 1985); Dowling (1988); Sudarsan et al. (1997). The cylinder is modelled as a beam (with flexural rigidity set to zero in some cases), coupled with the fluid dynamics via inviscid terms (Lighthill 1960), friction and specific models of the flow-induced forces at the trailing end. Prediction of divergence and flutter using this model were found to be in good agreement with experiments in Semler et al. (2002). Further refinements, including accounting for nonlinear effects, showed that the linear approach was robust, at least for the first instabilities encountered: divergence occurs in a supercritical form and is followed by flutter. The flutter instability threshold computed on the deformed buckled solution was similar to that of the linear prediction, where it is considered as a loss of stability of the undeformed configuration (Semler et al. 2002).

The case of very long cylinders has been recognized in some of the earliest papers as being of particular interest (see Ortloff \& Ives 1969; Pao 1970; Lee 1981). This interest arose from practical applications where the length-to-diameter ratio $L / D$ would exceed $10^{2}$ or even $10^{3}$. The above authors, unfortunately using the uncorrected equations of motion, found that very long cylinders had a specific stability behaviour, which differed from that of shorter ones. The problem of divergence instability of cylinders with increasing length has been solved by Triantafyllou \& Chryssostomidis (1984). They found that, as the length is increased, divergence persists for streamlined cylinders, but disappears when they are blunt. Neglecting all flexural rigidity, Triantafyllou \& Chryssostomidis (1985) found that long cylinders should not flutter at all. Dowling (1988) reached the same conclusion using a more elaborate matched asymptotic expansion approach in which bending stiffness was taken into account where needed. Experiments on such long systems are somewhat inconclusive because of the difficulty of conducting accurate laboratory experiments with very slender beams in flow. Yet, divergence and flutter have been observed for cylinders where $L / D$ is larger than 100 by Ni \& Hansen (1978) and by Sudarsan et al. (1997), and in such cases the deformation seems to be confined to the downstream part of the cylinder.

The effect of length on stability of coupled fluidelastic slender systems is an important issue. In other similar systems that are tensioned by friction and/or gravity, several authors found the existence of a limit configuration for long lengths. Doare 
$\&$ de Langre (2002), considering a hanging fluid-conveying pipe tensioned by gravity, found in both experiments and numerical simulations that the flutter instability, equivalent to the classical garden-hose instability, was confined to a finite zone close to the downstream end. Provided the pipe was long enough to contain this zone, the instability was not affected by the length of the domain. A similar conclusion was reached for hanging ribbons with axial flow in Lemaitre, Hémon \& de Langre (2005), a filament inclined in the flow in Schouveiler et al. (2005), a filament in plane soap flow in Zhang et al. (2000), and a hanging beam with a follower force in de Langre, Doaré \& Pellet (2001).

Building on these results, we address in this paper the issue of flutter of long cylinders, where the meaning of 'long' has yet to be defined. In $\S 2$, the equations of motion are recalled and a new dimensionless form is proposed, which is appropriate for the analysis of the effect of the length. The procedure used for their solution is also described. In $\S 3$, the effect of length on stability is analysed. In $\S 4$, the case of very long cylinders is considered, and a link is established with previous analyses, particularly when the cylinder is modelled as a string. Comparison with experiments is also presented. Finally, in $\S 5$, we propose a simple model for the dynamics of these long cylinders subjected to flow, using data on short cylinders.

\section{Equation of motion}

\subsection{A cantilever beam in axial flow}

Following Païdoussis (1973) and Païdoussis et al. (2002), the cylinder is modelled as a beam, i.e. with non-vanishing flexural rigidity. The equation governing the lateral motion $Y(X, T)$ reads

$$
E I \frac{\partial^{4} Y}{\partial X^{4}}-\frac{\partial}{\partial X}\left(\Theta \frac{\partial Y}{\partial X}\right)+m \frac{\partial^{2} Y}{\partial T^{2}}=F_{F},
$$

where $E I$ is the flexural rigidity, $\Theta(X)$ is the local axial tension, $m$ is the mass per unit length, and $F_{F}$ is the transverse fluid force per unit length acting on the beam. A uniform axial flow is assumed. The fluid loading resulting from the motion may be modelled as the sum of the following:

(a) an inviscid force as in Lighthill (1960), acting on the entire length of the cylinder, that depends on the local deformation

$$
\boldsymbol{F}_{I}=-\rho A\left(\frac{\partial^{2} Y}{\partial T^{2}}+2 U \frac{\partial^{2} Y}{\partial X \partial T}+U^{2} \frac{\partial^{2} Y}{\partial X^{2}}\right) \boldsymbol{e}_{Y},
$$

where $\rho$ is the fluid density, $A=\pi D^{2} / 4$ is the cross-sectional area of the cylinder, $U$ is the mean flow velocity and $\boldsymbol{e}_{Y}$ is the transverse unit vector; this expression includes added mass, added stiffness (centrifugal load) and Coriolis effects;

(b) a drag force with normal and tangent components acting on the entire length of the cylinder,

$$
\boldsymbol{F}_{D}=-\frac{1}{2} \rho D U C_{N}\left(\frac{\partial Y}{\partial T}+U \frac{\partial Y}{\partial X}\right) \boldsymbol{n}+\frac{1}{2} \rho D U^{2} C_{T} \boldsymbol{t},
$$

where $\boldsymbol{n}$ and $\boldsymbol{t}$ are the local normal and tangential unit vectors of the deformed beam axis (figure 1) and $C_{N}, C_{T}$ are the normal and tangential drag coefficients, respectively; a detailed discussion of the relation of the latter to the cylinder roughness may be found in Païdoussis (2003), but suffice it to say here that they are of the same order 
of magnitude, typically 0.01 , and that values of the ratio $C_{N} / C_{T}$ of 0.5 and 1.5 correspond, respectively, to rough and smooth cylinders;

(c) a base drag force

$$
\boldsymbol{F}_{B D}=\frac{1}{2} \rho D U C_{b} \boldsymbol{t},
$$

where $C_{b}$ typically varies from zero, for a streamlined end, to $\pi / 4$ for a blunt end;

(d) a lift force exerted at the downstream end

$$
\boldsymbol{F}_{L}=f \rho A U\left(\frac{\partial Y}{\partial T}+U \frac{\partial Y}{\partial X}\right) \boldsymbol{n},
$$

where $f$ depends on the shape of this end, typically being zero for a blunt end and unity for a perfectly streamlined end.

Including the effect of all these forces yields

$$
\begin{aligned}
E I \frac{\partial^{4} Y}{\partial X^{4}}-\frac{\partial}{\partial X}(\Theta & \left.\frac{\partial Y}{\partial X}\right)+\frac{1}{2} \rho U^{2} D\left(C_{N}-C_{T}\right) \frac{\partial Y}{\partial X} \\
& +\frac{1}{2} \rho U D C_{N} \frac{\partial Y}{\partial T}+2 \rho A U \frac{\partial^{2} Y}{\partial X \partial T}+(m+\rho A) \frac{\partial^{2} Y}{\partial T^{2}}=0,
\end{aligned}
$$

where the tension is given by

$$
\Theta(X)=-\rho U^{2}\left(A-\frac{1}{2} D^{2} C_{b}+\frac{1}{2} D C_{T} X\right) .
$$

The tension $\Theta(X)$ includes drag forces and the coefficient of the centrifugal load, (2.2), which has the same effect on the dynamics of the cylinder as an axial compressive force of magnitude $\rho A U^{2}$. We shall refer to this force as the 'effective compressive force'.

It is emphasized here that in this paper, unlike in many previous studies, $X=0$ is at the equilibrium position of the downstream end of the body, while the upstream end is at $X=-L$ (figure 1). Hence, the corresponding boundary conditions at the upstream and downstream ends, respectively, read

$$
\left.\begin{array}{l}
Y(-L)=\frac{\partial Y}{\partial X}(-L)=0, \\
\frac{\partial^{2} Y}{\partial X^{2}}(0)=\left[E I \frac{\partial^{3} Y}{\partial X^{3}}+f \rho A U\left(\frac{\partial Y}{\partial T}+U \frac{\partial Y}{\partial X}\right)\right](0)=0 .
\end{array}\right\}
$$

These equations have been used in various forms by Triantafyllou \& Chryssostomidis (1985), Dowling (1988), Sudarsan et al. (1997) and Païdoussis et al. (2002), with some simplifying assumptions as discussed in Païdoussis (2003). More sophisticated models including lateral confinement or tapering effects are also given in this same reference.

\subsection{Neutral point}

It appears in (2.7) that, at the location $X=X_{C}=-L_{C}$ where

$$
L_{C}=\frac{D\left(\pi-2 C_{b}\right)}{2 C_{T}},
$$

the local tension $\Theta$ vanishes, as the tension induced by friction and by the base drag is exactly balanced by the effective compressive force resulting from the centrifugal load. Downstream of this point, $-L_{C}<X \leqslant 0$, the beam is in compression, and upstream of this point, $X<-L_{C}$, it is in tension. The parameters $X_{C}$ and $L_{C}$ will now be referred to as the neutral point and the compression length, respectively. As noted 
by Triantafyllou \& Chryssostomidis (1985) and Dowling (1988), this particular point is of interest because the only stiffness arises from the flexural rigidity. Therefore, a special treatment is necessary when a string approximation is used, that is when all flexural rigidity is neglected. In fact, with all points downstream of the neutral point being in compression, no stiffness exists in this segment of the cylinder other than that due to flexural rigidity. Using typical values of the coefficients $C_{b}$ and $C_{T}$, the order of magnitude of $L_{C} / D$ is about $10^{2}$. Therefore, for most applications in the field of towed systems the length $L$ will be larger than $L_{C}$ so that a neutral point will exist. It should be noted that the position of this neutral point does not depend on the flow velocity, contrary to the cases where the load balance that defines the neutral point involves gravity, as in Doaré \& de Langre (2002) and Lemaitre et al. (2005).

Perhaps the existence of a compression over a portion of the cylinder, downstream of the thereby defined neutral point, is counterintuitive. The analogy between internal and external axial flow over the cylinder may be invoked here to make this matter physically clearer. Consider internal flow of fluid mass per unit length $M$ and velocity $U$ in a cantilevered blunt-ended flexible cylinder. Equation (2.2) applies in this case also, with $M$ replacing $\rho A$; yet in this case it is easier to visualize that at the free end there exists an effective compressive load equal to $M U^{2}$ (Païdoussis 1998). By similarity (see Païdoussis 2003 for a more extensive discussion) there is a similar (effective) compressive force at the end of a blunt cantilevered cylinder, $\rho A U^{2}$. Moreover, for external viscous flow, there is a base drag as given by (2.4) and a distributed frictional drag as given by the second term of (2.3), generating tensile forces. It is the balance of these tensile and compressive forces that gives rise to a point where the tension is null, and downstream of it, the tension is effectively replaced by compression.

\subsection{Dimensionless forms}

Following Païdoussis et al. (2002), we define the dimensionless variables

$$
x=\frac{X}{L}, \quad \eta=\frac{Y}{L}, t=\left(\frac{E I}{m+\rho A}\right)^{1 / 2} \frac{1}{L^{2}} T, u=\left(\frac{\rho A}{E I}\right)^{1 / 2} U L, \beta=\frac{\rho A}{m+\rho A}, \varepsilon=\frac{L}{D} .
$$

For convenience, we also introduce the coefficients $c_{T}=4 C_{T} / \pi, c_{N}=4 C_{N} / \pi, c_{b}=$ $4 C_{b} / \pi$, so that $L_{C}=D\left(2-c_{b}\right) / c_{T}$. Equation (2.6) may be re-written in dimensionless form as

$$
\begin{aligned}
\frac{\partial^{4} \eta}{\partial x^{4}}+\frac{\partial}{\partial x}\left[u ^ { 2 } \left(1-\frac{1}{2} c_{b}\right.\right. & \left.\left.+\frac{1}{2} \varepsilon c_{T} x\right) \frac{\partial \eta}{\partial x}\right]+\frac{\varepsilon c_{T}}{2}\left(\frac{c_{N}}{c_{T}}-1\right) u^{2} \frac{\partial \eta}{\partial x} \\
& +\frac{\varepsilon c_{T}}{2}\left(\frac{c_{N}}{c_{T}}\right) \sqrt{\beta} u \frac{\partial \eta}{\partial t}+2 \sqrt{\beta} u \frac{\partial^{2} \eta}{\partial t \partial x}+\frac{\partial^{2} \eta}{\partial t^{2}}=0
\end{aligned}
$$

with the boundary conditions

$$
\left.\begin{array}{l}
\eta(-1)=\frac{\partial \eta}{\partial x}(-1)=0, \\
\frac{\partial^{2} \eta}{\partial x^{2}}(0)=\left[\frac{\partial^{3} \eta}{\partial x^{3}}+f u\left(\sqrt{\beta} \frac{\partial \eta}{\partial t}+u \frac{\partial \eta}{\partial x}\right)\right](0)=0 .
\end{array}\right\}
$$

This dimensionless set of equations is identical to that used in Païdoussis et al. (2002), except for the change of variables $x=\xi-1$. The neutral point is located at 
$x_{C}=-L_{C} / L=-\left(2-c_{b}\right) / \varepsilon c_{T}$. Short cylinders may be defined by the condition that $\varepsilon c_{T} \ll 1$, so that all length-dependent terms vanish in (2.11).

In order to analyse the effect of length on stability, a more appropriate dimensionless form may be defined, where the compression length $L_{C}$ is used in the scaling in place of the cylinder length $L$, as in Doare \& de Langre (2002) and Lemaitre et al. (2005). The corresponding variables are

$$
\left.\begin{array}{l}
z=\frac{X}{L_{C}}, \quad y=\frac{Y}{L_{C}}, \quad \tau=\left(\frac{E I}{m+\rho A}\right)^{1 / 2} \frac{1}{L_{C}^{2}} T, \\
v=\left(\frac{\rho A}{E I}\right)^{1 / 2} U L_{C}, \quad \beta=\frac{\rho A}{m+\rho A}, \quad \ell=\frac{L}{L_{C}} .
\end{array}\right\}
$$

Equation (2.6) now becomes

$$
\begin{aligned}
\frac{\partial^{4} y}{\partial z^{4}}+\frac{\partial}{\partial z}\left[v^{2}\left(1-\frac{1}{2} c_{b}\right)(1+z) \frac{\partial y}{\partial z}\right] & +v^{2}\left(1-\frac{1}{2} c_{b}\right)\left(\frac{c_{N}}{c_{T}}-1\right) \frac{\partial y}{\partial z} \\
& +\sqrt{\beta} v\left(1-\frac{1}{2} c_{b}\right) \frac{c_{N}}{c_{T}} \frac{\partial y}{\partial \tau}+2 \sqrt{\beta} v \frac{\partial^{2} y}{\partial \tau \partial x}+\frac{\partial^{2} y}{\partial \tau^{2}}=0
\end{aligned}
$$

and the corresponding boundary conditions are

$$
\left.\begin{array}{l}
y(-\ell)=\frac{\partial y}{\partial x}(-\ell)=0 \\
\frac{\partial^{2} y}{\partial z^{2}}(0)=\left[\frac{\partial^{3} y}{\partial z^{3}}+f v\left(\sqrt{\beta} \frac{\partial y}{\partial \tau}+v \frac{\partial y}{\partial z}\right)\right](0)=0 .
\end{array}\right\}
$$

In this dimensionless form, the effect of length $\ell$ now appears only in the upstream boundary conditions. The new variables are related to the previous set by $z=x \ell$, $\tau=t \ell^{2}, v=u / \ell$ and $\ell=\varepsilon c_{T} /\left(2-c_{b}\right)$. The neutral point is now located at $z=-1$, and long cylinders may be defined by the condition that $\ell \gg 1$. Note that other dimensionless forms, such as one using $D$ as a length scale as in Triantafyllou \& Chryssostomidis $(1984,1985)$, would also allow us to analyse the dependence of the dynamics on $L$.

\subsection{Solution procedure}

To analyse the stability of the straight-line position of the cylinder, perturbations of the form $y(x, t)=\varphi(x) \mathrm{e}^{\mathrm{i} \omega t}$, in $(2.11)$, or equivalently $y(z, t)=\psi(z) \mathrm{e}^{\mathrm{i} \Omega \tau}$, in $(2.14)$, are imposed. The corresponding equation for the mode shape $\varphi(x)$ reads

$\varphi^{(4)}+u^{2}\left(1-\frac{1}{2} c_{b}+\frac{1}{2} \varepsilon c_{T} x\right) \varphi^{(2)}+\left(\frac{1}{2} \varepsilon c_{N} u^{2}+2 \mathrm{i} \omega \sqrt{\beta} u\right) \varphi^{\prime}+\left(\frac{1}{2} \mathrm{i} \omega \varepsilon c_{N} \sqrt{\beta} u-\omega^{2}\right) \varphi=0$,

with $\varphi(-1)=\varphi^{\prime}(-1)=\varphi^{(2)}(0)=\left[\varphi^{(3)}(0)+f u\left(\mathrm{i} \omega \sqrt{\beta} \varphi(0)+v \varphi^{\prime}(0)\right)\right]=0$, where ()$^{(n)}$ denotes differentiation with respect to $x$. Alternatively, using the other set of dimensionless variables, we obtain

$$
\begin{aligned}
\psi^{(4)}+v^{2}\left(1-\frac{1}{2} c_{b}\right)(1+z) \psi^{(2)}+ & {\left[v^{2}\left(1-\frac{1}{2} c_{b}\right) \frac{c_{N}}{c_{T}}+2 \sqrt{\beta} \mathrm{i} \Omega v\right] \psi^{\prime} } \\
+ & {\left[\mathrm{i} \Omega \sqrt{\beta} v\left(1-\frac{1}{2} c_{b}\right) \frac{c_{N}}{c_{T}}-\Omega^{2}\right] \psi=0, }
\end{aligned}
$$

with $\psi(-\ell)=\psi^{\prime}(-\ell)=\psi^{(2)}(0)=\left[\psi^{(3)}(0)+f v\left(\mathrm{i} \Omega \psi(0)+v \psi^{\prime}(0)\right)\right]=0$, where differentiation is now with respect to $z$.

For a given set of parameters, say $\left(u, \varepsilon c_{T}, c_{N} / c_{T}, c_{b}, f, \beta\right)$ or equivalently $(v, \ell$, $\left.c_{N} / c_{T}, c_{b}, f, \beta\right)$, the eigenmodes $\left(\varphi_{N}, \omega_{N}\right)$ or $\left(\psi_{N}, \Omega_{N}\right)$ may be computed by solving 

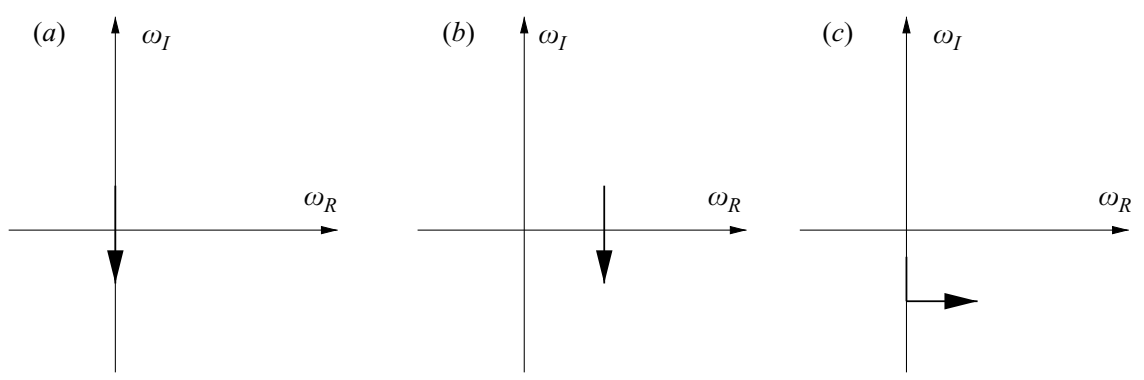

FIGURE 2. Types of instability, depending on the crossing of axes in the complex $\omega$-plane.

(a) Divergence, (b) flutter by Hopf bifurcation, $(c)$ flutter of the Païdoussis type.

a standard linear eigenvalue problem. Stability is ensured when all eigenfrequencies, $\omega$ or $\Omega$, have a positive imaginary part. When one of the imaginary parts becomes negative while the real part of the frequency is equal to zero, divergence results. Conversely, an instability associated with a positive real frequency is defined as flutter. It has been found necessary for fluidelastic systems to distinguish between two kinds of flutter: if an eigenvalue crosses the real axis, $\omega_{I}=0$, into the flutter domain defined by $\omega_{I}<0$, a classical Hopf bifurcation occurs. Alternatively, when an eigenvalue emerges from the imaginary axis, $\omega_{R}=0$, into the flutter domain, the instability is commonly referred to as Païdoussis flutter, as originally proposed by Done \& Simpson (1977). For this particular form of flutter, which occurs only after divergence, oscillations of increasing frequency occur as the control parameter, in our case velocity, is increased.

The two types of flutter are illustrated in figure 2. As Païdoussis flutter is a secondary instability derived from an analysis based on the initial geometric configuration rather than the post-divergence configuration, it may be irrelevant. Yet, nonlinear computations in Semler et al. (2002) have shown that the linear analysis gives a good approximation of the qualitative and quantitative set of instabilities for short cylinders. As we seek to explore systematically the effects of several parameters on stability, the simpler linear approach is used in this paper, with a nonlinear computation on a particular case, $\S 3.4$, to check its relevance in our case of long cylinders.

A Galerkin method based on the eigenmodes of a cantilevered beam without flow has been used for similar types of equations pertaining to the linear stability of fluid-conveying pipes, plates or cylinders in axial flow, as shown in Païdoussis (1998, 2003). In terms of the numerical method, it has been observed that, as the length of the system (parameter $\varepsilon c_{T}$ ) is increased, more and more modes are required in order to achieve acceptable convergence of the eigenmode analysis. For instance, for a fluid-conveying pipe tensioned by gravity (Doaré \& de Langre 2002) or for a hanging ribbon (Lemaitre et al. 2005), it was found that up to fifty modes were required. This could be understood by considering that an increasing number of modes play a role in the instability as the length $L$ is increased: the zone where the corresponding deformation takes place is almost of constant size and therefore diminishes when scaled by the length of the system, so that modes of shorter wavelength, and therefore higher order, are required in the modal basis.

We use here a standard finite-difference scheme in space as in Sugiyama \& Kawagoe (1975) to discretize (2.17). It is thus easier to adapt the spatial discretization to the relevant length scales. Unless otherwise specified, our results are obtained using 300 nodes. A more general approach using finite elements may be found in Bhattacharyya, Vendhan \& Sudarsan (2000). 


\section{Effect of length on stability}

\subsection{Previous results}

We summarize some results obtained by Semler et al. (2002), where equation (2.16) has been solved for several values of $f, c_{N} / c_{T}, \varepsilon c_{T}$, with the mass parameter $\beta$ being fixed at 0.47 which is close to neutral buoyancy $(\beta=0.5)$. The relation between $c_{b}$ and $f$, the two coefficients related to the shape of the downstream end, was set to $c_{b}=1-f$. Note that the precise interdependence of $c_{b}$ and $f$ is not really known. However, it is clear that as the end of the cylinder becomes more streamlined ( $f$ tends towards unity), the base drag is diminished (and in the limit $c_{b}$ tends to zero). Vice versa, as the free end becomes blunt, $f$ comes close to 0 and $c_{b}=O(1)$. For simplicity, therefore, the linear relationship $c_{b}=1-f$ was used by Païdoussis (2003) and has been adopted here also.

Computations showed that the critical velocities that yielded either divergence or flutter both increased with length, by varying the dimensionless parameter $\varepsilon c_{T}$. Depending on the values of $f$ and $c_{N} / c_{T}$, however, instabilities could cease to exist above a finite length of the cylinder. Flutter appeared mostly in the form of a Hopf bifurcation, but the possibility of Païdoussis type flutter was mentioned, and calculated in one case. As noted by these authors and confirmed by recent computations, convergence of the results was not always established for the longer cylinders, typically from $\varepsilon c_{T}=1$ up to the highest tested value, $\varepsilon c_{T}=4$. For instance, for $f=0.5$, flutter was found for $\varepsilon c_{N}$ up to 3 , that is $\ell=2$, but these results were later found to display an artificial convergence: our new calculations using the same Galerkin procedure showed that the corresponding critical velocity increases steadily with the number of modes, without reaching convergence.

Note that in terms of predicting the effect of the dimensional length $L$ on a dimensional critical velocity $U$, the apparent increase of the dimensionless velocity $u$ with the dimensionless length $\varepsilon c_{T}$ can be misleading, as both are defined using $L$. Using the proposed dimensionless velocity $v=u / \ell$, which does not involve $L$ but $L_{C}$, is more appropriate for studying the effect of length. If the results are expressed in terms of $(v, \ell)$ instead of $\left(u, \varepsilon c_{T}\right)$, the highest value of $\ell$ considered is $\ell \simeq 2$ which does not allow us to draw conclusions for truly long cylinders, $\ell \gg 1$. Similarly, the experimental results of Païdoussis (1966), where $\varepsilon$ is varied up to a value of 45 and affects stability, do not come into the range of long cylinders in our sense, since $\ell<2$.

The results presented next explore this range $(\ell \gg 1)$ using the numerical procedure described above to solve (2.17), which is the adequate dimensionless form for long cylinders. We first present two cases which are representative of the results to be presented in more detail in subsequent sections.

\subsection{A system stabilized at sufficiently large $\ell ; f=0.5$}

The case of $f=0.5$, which is that of a rather blunt end, such as a hemispherical one, is analysed for $c_{N} / c_{T}=1$, which corresponds to a medium surface roughness. For a given value of $\ell$ the velocity $v$ is varied, and the domains of instability as well as the type of instability are determined in the $(\ell, v)$-plane, solving (2.17) directly. Figure 3(a) shows the results obtained with the procedure described above and their comparison with previous computations using a Galerkin approximation of the same equations. For the divergence instability the critical velocity is found to decrease steadily with length, up to about $\ell=1$. This evolution may be compared to the result given by Semler et al. (2002) in terms of the dependence of $u$ on length: for $\varepsilon c_{N}=0$ they found that divergence occurred at $u=2.39$ which yields the asymptotic relation for small 

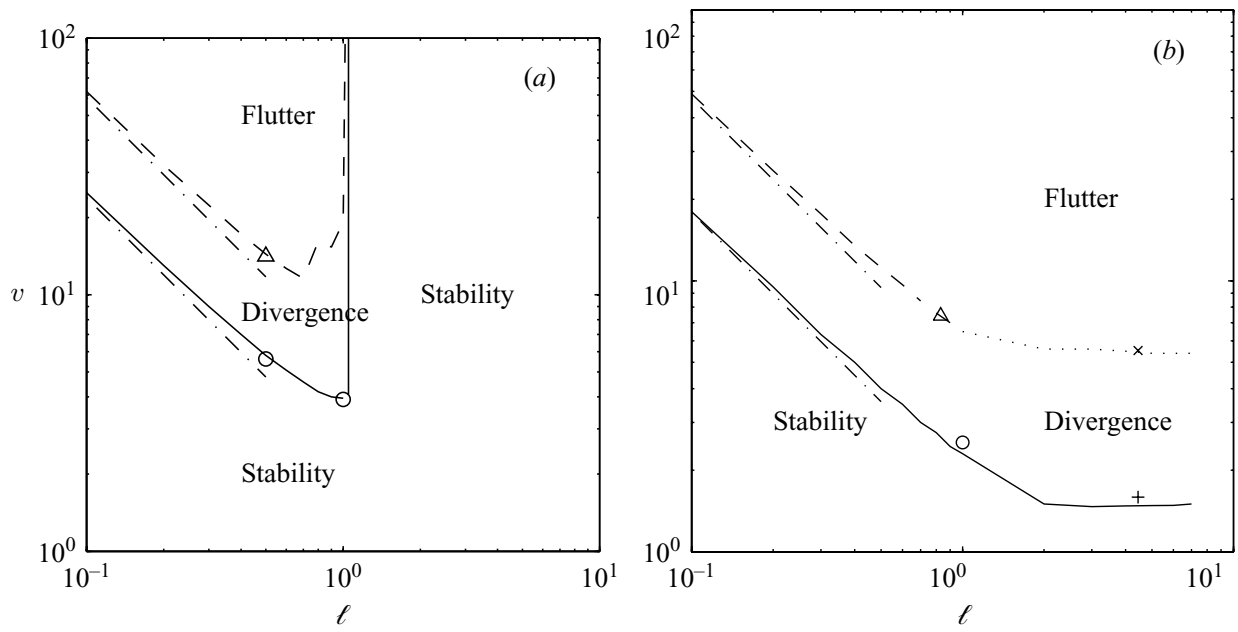

FiguRE 3. Effect of length on the stability of a cylinder, $(a)$ for a system stable at high enough $\ell$, with $f=0.5,(b)$ for one unstable at high $\ell$, with $f=0.8$. Present calculations: - critical velocity for divergence; - --, critical value for flutter by Hopf bifurcation; ..., critical velocity for flutter of the Païdoussis type. Results derived from the calculations by Semler et al. (2002): $-\cdot-$, limits for divergence and flutter of short cylinders; $\bigcirc, \Delta$, for cylinders of intermediate length; $\times,+$, new calculations using the Galerkin method.

cylinders, $v=2.39 / \ell$, which compares well with our results. Near $\ell=1$, divergence disappears in our case, which is consistent with their results at $\varepsilon c_{N}=1.5$.

Flutter is also found above the divergence limit. The evolution of the critical velocity is similar to that of divergence: for short cylinders, $\ell \ll 1$, it is consistent with their results at $\varepsilon c_{N}=0$, yielding $v=5.87 / \ell$. Flutter is suppressed above $\ell=1$.

\subsection{A system not stabilized for large $\ell ; f=0.8$}

Considering now a cylinder with $f=0.8$, i.e. with a well streamlined end, yields distinct results depending on the length $\ell$, figure $3(b)$. For short cylinders, $\ell \ll 1$, the divergence and flutter limits decrease with $\ell$ in a manner similar to that of the previous case, as could be predicted from the results of Semler et al. (2002) $(v=1.79 / \ell$ for divergence, $v=4.72 / \ell$ for flutter). However, for long cylinders, divergence and flutter persist, in contrast to the previous case with $f=0.5$. Moreover, the corresponding thresholds are found to depend weakly on $\ell$. Both divergence and flutter results for long cylinders are confirmed by converged Galerkin computations at $\ell=4.4$ using 12 modes. Note that above $\ell=1$, flutter becomes of the Païdoussis type as was hypothesized by Semler et al. (2002).

In figure 4 , we show the shape of the unstable mode, $\psi(z)$, for several values of the cylinder length. The eigenmodes are of arbitrary amplitude. The divergence deformation, figure $4(a-c)$, closely resembles that of a first mode divergence and is clearly confined to the downstream end of the cylinder, as was previously reported by Triantafyllou \& Chryssostomidis (1984). The flutter mode shape is evaluated at a velocity $v$ which is $10 \%$ above the critical value, and is shown by reconstructing the motion at several instants of a cycle, but neglecting the imaginary component (growth rate). The fluttering motion, figure $4(d-e)$, is also confined to a region of dimensionless scale 1 , which corresponds to $L_{C}$ in dimensional terms. Note that the fluttering motion is similar for the Hopf bifurcation case, $\ell=0.5$, and the Païdoussis type flutter cases, $\ell=2$ and 5 . 
(a)

(b)

(c)

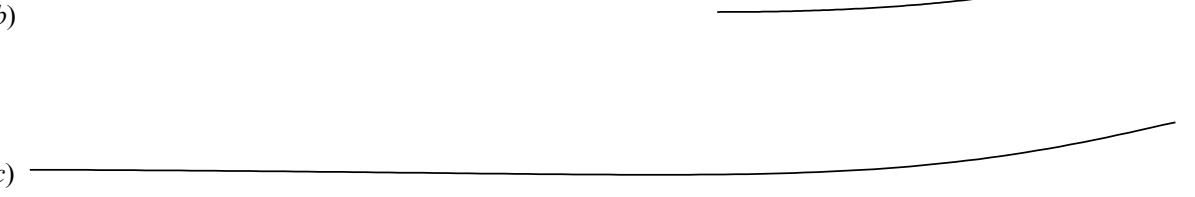

(d)

(e)

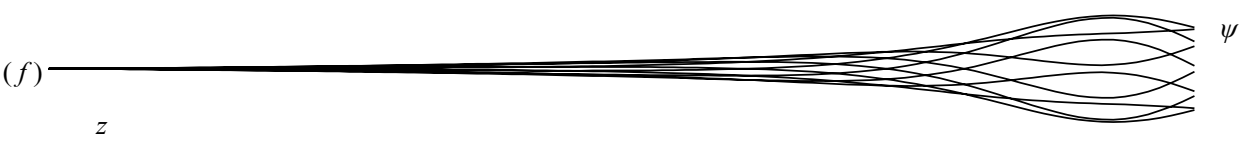

Figure 4. Modal shape $\psi(z)$ of the unstable modes as a function of the length of the cylinder for $f=0.8$. $(a-c)$ Divergence mode shape for $\ell=0.5,2,5$, respectively. $(d-f)$ Flutter motion over a cycle of oscillations reconstructed from the complex mode shape at $v=1.1 v_{F}$ for $\ell=0.5,2,5$, respectively.

These results confirm the existence of limit regimes for long cylinders where divergence and flutter exist and where the corresponding motion is limited to a downstream region of typical size $L_{C}$. This is consistent with previous work on hanging fluid-conveying pipes (Doaré \& de Langre 2002) and on ribbons subjected to flow (Lemaitre et al. 2005).

\subsection{Nonlinear model}

As noted before, because flutter arises following divergence, a nonlinear stability analysis on the deformed configuration is required. In this problem, the dominant nonlinear terms are associated with geometric effects, when the fluid forces are applied on the instantaneous deformed position of the cylinder. The corresponding set of equations has been derived by Lopes, Païdoussis \& Semler (2002).

Using a Galerkin approximation of the displacement of the cylinder, these coupled equations were solved in Semler et al. (2002) for relatively short cylinders, $\varepsilon=0.5$ with $f=0.7$, so that $\ell=0.29$. It was found that stability is first lost by divergence, followed by restabilization and then flutter. A rich sequence of bifurcations then occurs, which is not discussed here. The critical velocities for divergence and flutter as well as the mode shapes compared well with experimental results. Moreover, the sequence of divergence and flutter was qualitively and quantitatively similar to that predicted by the linear equations.

We use in our approach exactly the same set of nonlinear equations of motion and the same numerical method (AUTO, Doedel \& Kernéves 1986), but with a high 

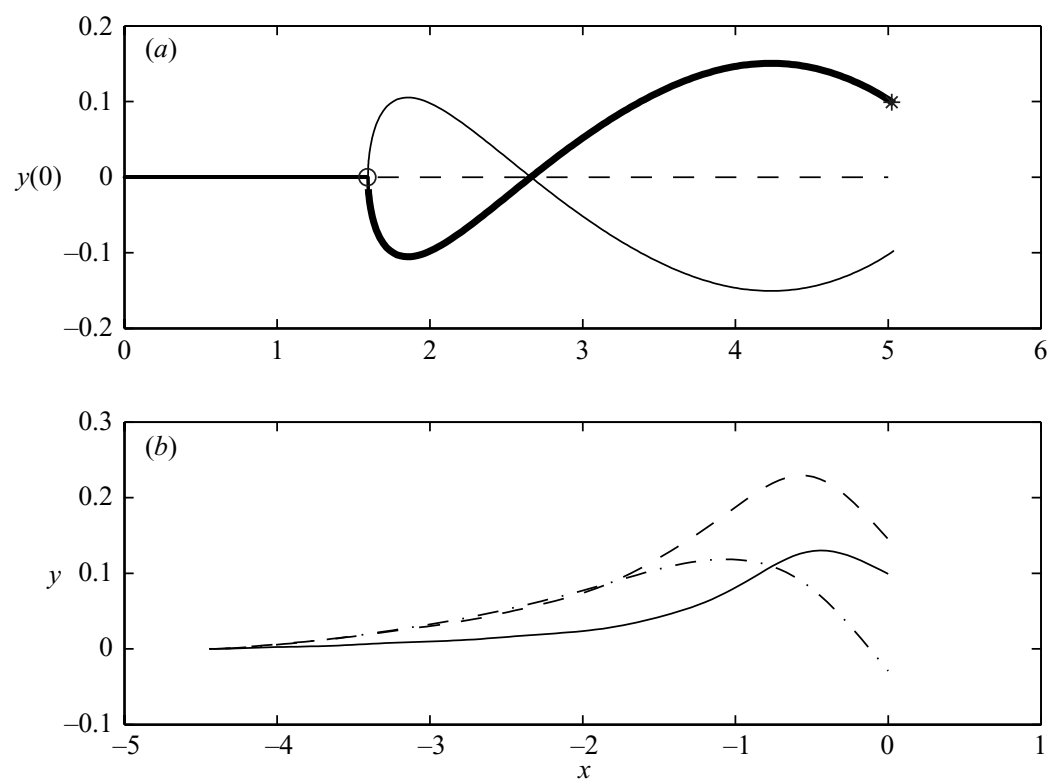

FIGURE 5. Solution of the nonlinear equation of motion for a long cylinder, $\ell=4.44, f=0.8$. (a) Bifurcation diagram of the displacement at the downstream end when the flow velocity is varied. o, Divergence at $v=1.59$; *, flutter at $v=5.03$. (b) Deformation of the cylinder for three flow velocities along the branch in bold line: $-\cdot-, v=2.5 ;---, v=3.5 ;-, v=5$.

number of modes $(N=12)$, for a system with $\varepsilon c_{N}=8$, corresponding to $\ell=4.44$ in our variables, and $f=0.8$. The resulting bifurcation diagram is shown in figure $5(a)$ in terms of the effect of the flow velocity $v$ on the dimensionless transverse displacement at the downstream end $y(0)$. The system is stable at low flow velocities, and the first bifurcation is a pitchfork bifurcation leading to divergence (buckling), occurring at $v=1.59$ which, as expected, is identical to the prediction of the linear analysis using the same Galerkin discretization. The bifurcation is supercritical resulting in a progressive deformation of the cylinder, as exemplified in figure $5(b)$ for several values of the velocity after divergence and before flutter. Note that the deformation in this range of velocities remains small, of the order of 0.1 , and becomes increasingly confined in the downstream region.

At $v=5.03$, the buckled shape loses stability via a Hopf bifurcation and flutter results. This critical value is reasonably close to the prediction of the linear stability analysis, which is $v=5.52$. In fact, the deformed static configuration just before the onset of flutter does not deviate significantly from the original straight shape, assumed in the linear analyis. This shows that the linear approach has some relevance, at least up to this second bifurcation.

\section{Stability of long cylinders}

\subsection{Stability domain}

The linear stability of long cylinders is now systematically explored by solving (2.17) for the length equal to $\ell=10$, since computations for longer cylinders showed small variations in the results. The corresponding discretization grid needed to ensure that about 30 nodes lie in the compression zone, of size 1 , is thus $N=300$. This allows an accurate representation of the gradients in that zone. The ratio $c_{N} / c_{T}$ is fixed at 1 , 


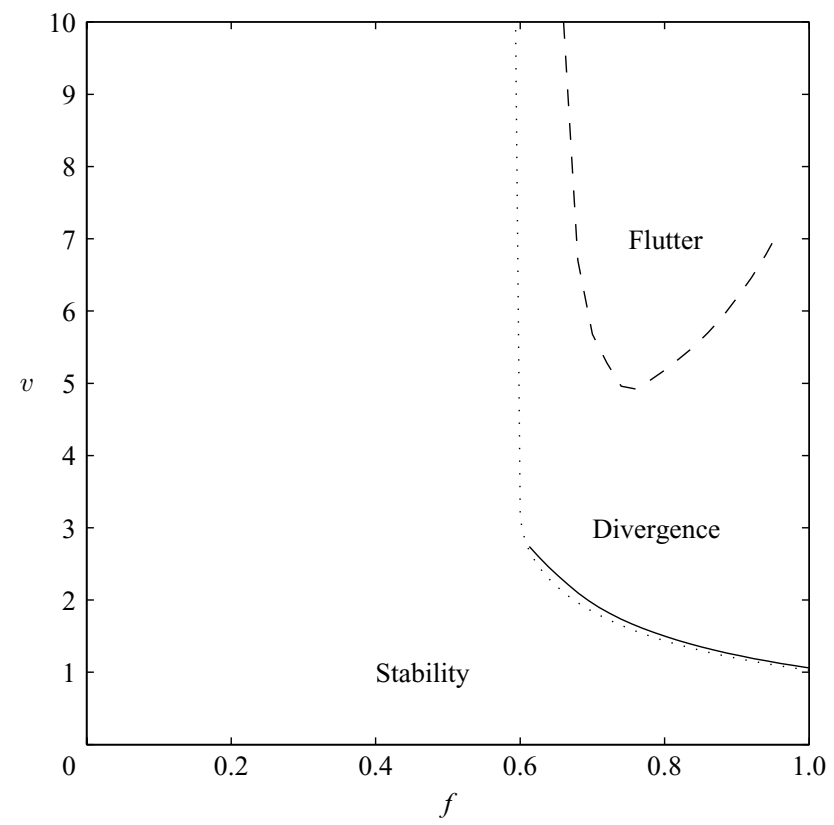

FIGURE 6 . Stability diagram for long cylinders, $\ell \gg 1$, from computations at $\ell=10$, depending on the parameter $f$ relative to the shape of the downstream end. - - Divergence limit; ---, flutter limit; ..., exact divergence limit adapted from the solution of Triantafyllou \& Chryssostomidis (1984), using (4.2) with the change of variables of (4.3).

unless otherwise specified. The parameter $f$ is varied from $f=0$ (blunt end) to $f=1$ (perfectly streamlined end).

Figure 6 shows the stability diagram in the parameter space $(f, v)$. For $f$ less than 0.6, no instability is found. For $f$ larger than 0.6, divergence is observed above velocities of order 1. Flutter, always of the Païdoussis type, arises approximately in the range $0.65<f<0.95$, for velocities of order 5. Calculations for values of $f$ close to 0.6 or to 1 converged with respect to both length and the number of nodes, and the corresponding velocities were clearly much higher than 5 . By varying the ratio $c_{N} / c_{T}$ from 0.5 to 1.5 , corresponding, respectively, to rough and smooth cylinders, it is found that the divergence and flutter domains always exist in the general form shown in figure 6, but with lower limits of $f$ that vary from 0.3 to 0.85 .

\subsection{Divergence}

Setting the frequency $\Omega$ to zero in (2.17), with $c_{N} / c_{T}=1$ yields

$$
\psi^{(4)}+v^{2}\left(1-\frac{1}{2} c_{b}\right)\left[(1+z) \psi^{\prime}\right]^{\prime}=0
$$

with the condition at the dowsntream end $\psi^{(3)}(0)+f v^{2} \psi^{\prime}(0)=0$; the other boundary conditions are omitted here for the sake of clarity. In the particular case where $c_{b}=0$, the exact solution for the critical velocity for divergence for a semi-infinite cylinder has been given by Triantafyllou \& Chryssostomidis (1984) in implicit form involving $v$ and $f$, namely

$$
\operatorname{Ai}^{\prime}\left(-v^{2 / 3}\right)-v^{2 / 3}(1-f)\left(\frac{1}{3}-\int_{0}^{-v^{2 / 3}} \operatorname{Ai}(s) \mathrm{d} s\right)=0,
$$

where $\mathrm{Ai}$ is the Airy function. It can be shown that no divergence occurs in the range of $f<0.75$. 
This solution may be generalized to include the effect of base drag, i.e. $c_{b} \neq 0$, by simply replacing $v$ and $f$ by $w$ and $g$ defined, respectively, by

$$
w^{2}=v^{2} /\left(1-\left(c_{b} / 2\right)\right) ; g=2 f /\left(2-c_{b}\right) .
$$

Equation (4.1) with the associated boundary condition then reduces to a form identical to one solved by Triantafyllou \& Chryssostomidis (1984), so that (4.2) may be used with $(w, g)$ in place of $(v, f)$ to derive the solution. If it is further assumed that $c_{b}=1-f$, the relation between $v$ and $f$ is fully determined. The range of divergence is $0.6<f<1$, and our numerical results are found to approximate this limit solution closely, as can be seen in figure 6 .

\subsection{Comparison with other models}

The existence of flutter for long cylinders, as found in our computations, seems to contradict previous results by Dowling (1988) and Triantafyllou \& Chryssostomidis (1985) who, using some simplifications on the role of bending stiffness, both concluded that long cylinders would not flutter.

The large length-to-diameter ratio in many practical applications of this problem have led several authors to develop solutions for the case where the bending stiffness of the cylinder is neglected. The simpler equation of motion then involves only secondorder, rather than fourth-order, derivatives in space. This equation is commonly associated with strings, a flexible medium with a stiffness that is due only to its tension. The works of Ortloff \& Ives (1969), Pao (1970), Lee (1981), Triantafyllou \& Chryssostomidis (1985) and Dowling (1988) use this approximation throughout or in parts of their models. A dimensionless number that would be appropriate for the comparison of the effect of bending and tension-induced stiffness is

$$
B=\frac{E I / \lambda^{4}}{\Theta_{0} / \lambda^{2}},
$$

where $E I$ is the bending stiffness, $\Theta_{0}$ is a tension to be defined and $\lambda$ is the scale of the spatial variations in $X$ of the displacement $Y$. It seems more appropriate to choose $\lambda=L_{C}$ than $\lambda=L$, as the deformation takes place on that scale (see figure 4). As the tension $\Theta(X)$ varies with both position and flow velocity, it becomes necessary to choose a particular value, say $\Theta_{0}$. With the main tensioning effect being axial friction and the scale of interest being $L_{C}$, we propose to use $\Theta_{0}=C_{T} \rho D U^{2} L_{C}$. The above dimensionless number is then approximately $B \simeq c_{T} / v^{2}$. Since the critical velocities found in our computations are of the order of $1-5$ and $c_{T}$ is of order $0.01, B$ is much smaller than unity. It may be expected that the role of the bending stiffness is important only in the compression region where no positive stiffness exists other than the one caused by bending.

In the work of Dowling (1988), a technique of matched asymptotic expansions is used to incorporate the bending stiffness only in the region where it plays a significant role, which is the region of large gradients in the vicinity and downstream of the neutral point. The corresponding developments require that $c_{N} / c_{T}$ be strictly less than 1. Applying this method to the case of $c_{N} / c_{T}=0.75$ and $\varepsilon c_{T}=60$, equivalent to $\ell$ in the range $30-60$ depending on the value of $f$, leads to the conclusion that no instability exists for any value of $v$ or $f$. The possibility of divergence is actually excluded in the development, as can be seen by setting the frequency to zero in the series solution. Yet, divergence has been known to exist based on the exact solution of Triantafyllou \& Chryssostomidis (1984), at least for $c_{N} / c_{T}=1$. Flutter found in our 

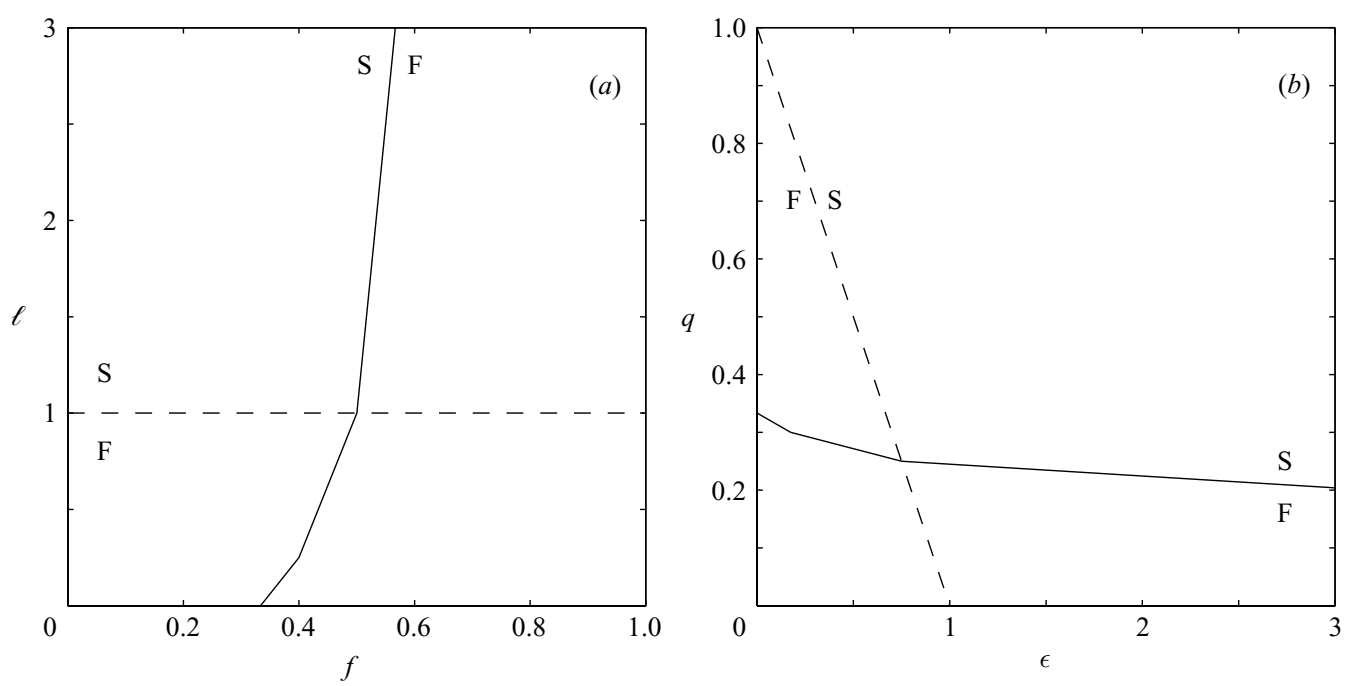

FIGURE 7. Stability diagrams for a string subjected to axial flow, $(a)$ in terms of our variables $(f, \ell),(b)$ in terms of the variables used by Triantafyllou \& Chryssostomidis (1985), $\epsilon=\ell(1+f) / 2, q=c_{b} / 2=(1-f) / 2$; - limit between stability and divergence for a beam at vanishing bending stiffness derived from our results; -.-, limit given by Triantafyllou \& Chryssostomidis (1985) using a string model. S, stability; F, flutter.

computations for long cylinders is always of the Païdoussis type and thus emanates from a divergent mode. We may therefore state that flutter is not found in Dowling's approach because, as divergence is not considered, it is consequently excluded from the analysis.

For the sake of comparison with the work of Triantafyllou \& Chryssostomidis (1985), where no bending stiffness is considered, our results may be used to derive the solution for a cylinder of vanishing stiffness. Letting EI go to zero is equivalent to considering the limit of stability for very high values of $v$ in graphs such as figure 3. The resulting stability diagram (figure $7 a$ ), gives regions of stability and flutter depending on the length $\ell$ and the coefficient $f$. For short strings, stability is ensured only for very blunt ends with $f<1 / 3$. As the length is increased, the domain of stability widens, but well streamlined ends still lead to flutter even for very long strings, as shown in figure 6.

We may compare these results with the criterion proposed by Triantafyllou \& Chryssostomidis (1985), using a pure string model and verified numerically by Bhattacharyya et al. (2000). Their criterion simply predicts instability when $q>1-\epsilon$ where $q=c_{b} / 2=(1-f) / 2$ and $\epsilon=\ell(1+f) / 2$. In terms of our variables this means that $\ell>1$, which differs significantly from our results (figure $7 a$ ). This comparison is also shown in terms of the variables $q$ and $\epsilon$ (figure $7 b$ ). The differences may be explained as follows. Their prediction of instability for all short strings, $\ell<1$, is directly associated with the absence of any positive tension in the system and, therefore, of any stiffness. In our computations, a bending rigidity, however small, is sufficient to stabilize short cylinders, provided the end is blunt enough (low values of $f$ ). Similarly, the prediction of stability for all long strings, $\ell>1$, is obtained, as noted by the authors, without particular treatment at the neutral point where some bending rigidity is required in order to ensure continuity. In our computations, we found that introducing this bending rigidity, even vanishingly small, does modify the 
stability of long cylinders, which are unstable provided the end is well streamlined. We may conclude that for this problem, the pure string model does not accurately predict the behaviour of cylinders, even of those with a very small bending rigidity.

\subsection{Comparison with experiments}

A detailed comparison with experiments exists in Païdoussis et al. (2002) and Semler et al. (2002), but only for short cylinders, i.e. with the dimensionless length $\ell$ always less than unity (see also Païdoussis 2003). Experiments in the regime of long cylinders are particularly difficult, as the length typically must be more than a hundred times larger than the diameter, with the friction coefficient $c_{T}$ of the order of 0.01 . The establishment of a steady axial flow with a straight initial configuration of a slender neutrally buoyant structure and the preservation of this state for a sufficient time to allow instabilities to develop are difficult to achieve. Tests by Ni \& Hansen (1978) on a flexible cable in a water tunnel and by Sudarsan et al. (1997) in a towing tank are the main existing sources.

In the early experiments by $\mathrm{Ni} \&$ Hansen (1978), the length-to-diameter ratio is $\varepsilon=L / D=500$. The measured friction coefficient is about $c_{T}=0.015$ and the hemispherical shape of the downstream end leads to approximately $f=0.5$. These parameters combine into a dimensionless length of $\ell=\varepsilon c_{T} /(1+f)=5$, which is not much larger than 1 , but already in the range of long cylinders, as can be seen from our results. Divergence, and some flutter near $u=30$ (corresponding to $v=6$ ), were observed. This is of the same order of magnitude as the critical velocities shown in figure 6, but for higher values of $f$ only. Ni \& Hansen (1978) also observed a deformation of about one diameter $(1.6 \mathrm{~cm})$ occurring in the last metre of the cylinder of total length $L=7.92 \mathrm{~m}$. This can be compared to the nonlinear results of figure 5 for $f=0.8$, where the deformation occurs over a dimensional length of order $L_{C}$, in our case with $L_{C}=1.52 \mathrm{~m}$, and a magnitude of order $0.1 L_{C}$, which is $15.2 \mathrm{~cm}$.

In the more recent experiments by Sudarsan et al. (1997), the largest length-todiameter ratio is $L / D=150$. A cylinder clamped at the upstream end with a well streamlined free end (referred to as test 20) is observed to diverge at a dimensionless velocity of $u=3.13$. When using the friction coefficient $c_{T}=0.0475$ measured by the authors and the value of $f=0.8$ corresponding to the well streamlined end, the dimensionless length is $\ell \simeq 4$ and therefore the experimental critical velocity is about $v \simeq 0.8$. This is of the same order of magnitude, though lower, than the divergence critical velocity for very long cylinders at $f=0.8$ (figure 6). It should nevertheless be noted that the values of $c_{T}$ obtained in these experiments are higher than those of other authors, as discussed in Païdoussis (2003). If a more typical value of $c_{T}=0.02$ is used, we obtain $\ell=1.7$ and $v=1.87$, which is located in the transition range between short and long cylinders (figure $3 b$ ). Results of other tests by Sudarsan et al. (1997), such as their tests 16 and 17 where a change of the upstream boundary conditions significantly modifies the stability, also suggest that the long cylinder regime is probably not reached for all these tests.

From these comparisons it may be stated that experimental evidence confirms the existence of instabilities for long cylinders, at flow velocities compatible with our predictions. Still, considerable work remains to be done to achieve the same accuracy in experimental comparisons for long cylinders as is available for short cylinders. Future work should include both detailed experiments and further parametric analysis of the model in both the linear and nonlinear regimes. 

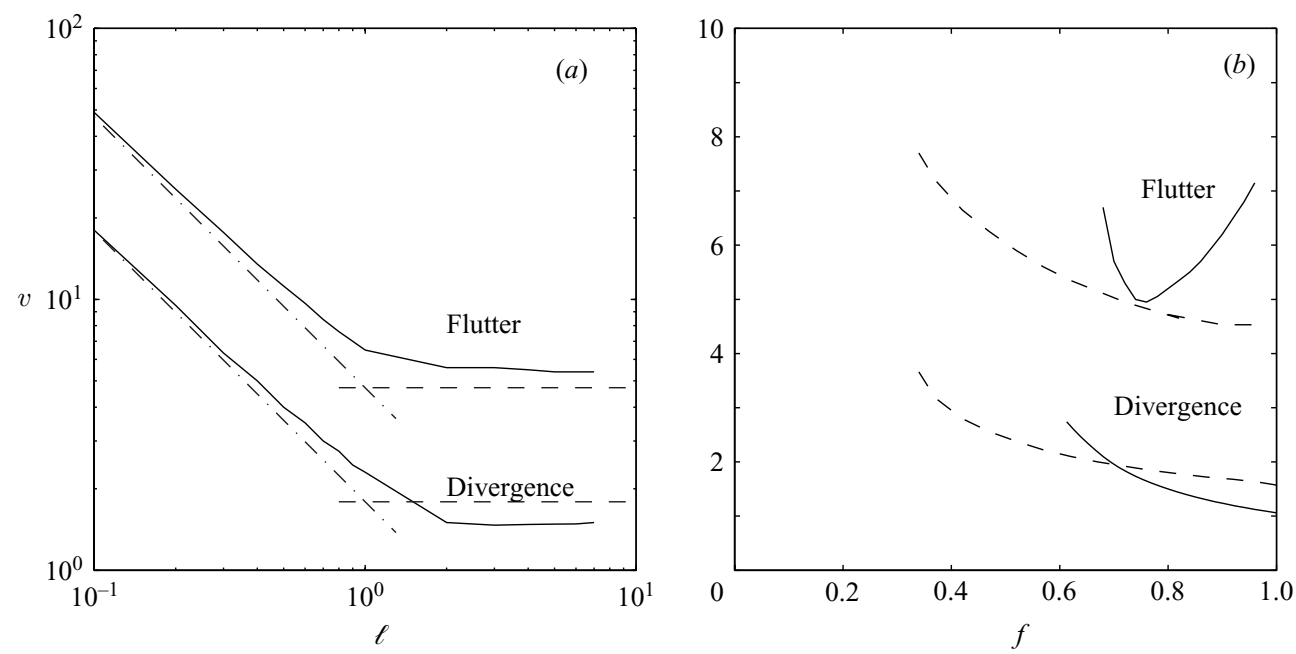

FIGURE 8. Model of the stability of a long cylinder using the stability diagram of a short cylinder of effective length $\ell=1$. (a) Effect of length on the critical velocities for divergence and flutter for $f=0.8 ;-$, present results; -- - , short cylinder approximation, equation (5.1); ---, long cylinder approximation, equation (5.2). (b) Stability diagram: ---, long cylinder approximation (5.2) for divergence and for flutter compared to the present results for long cylinders (-).

\section{A model for long cylinders using results for short cylinders}

For short cylinders, in the sense of $\varepsilon c_{T} \ll 1$, in (2.11) the critical velocity $u$ is independent of $\varepsilon c_{T}$ and depends only on $f$, for given values of $c_{N} / c_{T}$ and $\beta$. In terms of our dimensionless velocity $v$ based on $L_{C}$, we then have

$$
v(\ell, f)=u(f) / \ell \text {. }
$$

Conversely, for long cylinders, $\ell \gg 1$, we have shown that the deformation is confined to the downstream end in a region of dimensional size $L_{c}$, which is 1 in dimensionless form.

Ni \& Hansen (1978) noted that the experimental results for their long cylinders could be compared with the model of Païdoussis (1973) provided the length of the cylinder was replaced, in the formula, by that of the region where the deformation took place. In a related problem, Doare \& de Langre (2002) showed that the dynamics of long hanging pipes was controlled by the length of the domain where unstable bending waves exist. We may therefore assume that the behaviour of a long cylinder will be similar to that of a short cylinder of effective length $\ell_{e}=1$, so that the critical velocities for divergence and flutter read

$$
v(\ell, f)=u(f) / \ell_{e}=u(f) .
$$

These two approximations, (5.1) for short cylinders and (5.2) for long cylinders, are shown in figure $8(a)$ for the case of $f=0.8$. Here, $u(f)$ represents the critical velocities for divergence and for flutter for a short cylinder, in the sense of $\varepsilon c_{T} \ll 1$, such as computed in Semler et al. (2002) for instance. The approximation for long cylinders is found to be reasonably good, both for the flutter and the divergence limits, and the combination of the two limits gives a good estimate over all values of the length.

Considering now the entire range of $f$, figure $8(b)$ shows the comparison of the limit for long cylinders found in figure 6 with the one derived from (5.2). The order 
of magnitude of both divergence and flutter critical velocities is approximated well, but a difference can be observed concerning the effect of the lift parameter $f$ on stability: for short cylinders, divergence and flutter arise above $f=1 / 3$, whereas this limit is at $f=0.6$ for long cylinders. This may be explained qualitatively in terms of the balance of forces that cause divergence. For short cylinders, the stabilizing forces are the bending stiffness and base drag at the end, which varies as $c_{b} U^{2}=(1-f) U^{2}$. The destabilizing forces are the negative added stiffness, proportional to $U^{2}$ and the lift force at the downstream end, proportional to $f U^{2}$. The balance results in a criterion for divergence that depends both on $f$ and $U^{2}$. Increasing $f$ (thus increasing streamlining) is destabilizing as it reduces drag-induced tension, through $c_{b}$, and increases the downstream lift. Note also that in the case of no bending stiffness, $E I=0$, all forces are proportional to $U^{2}$ and the critical velocity cannot depend on $U$. In long cylinders, an additional stabilizing force is given by the axial friction, which plays a role only when $\ell=L / L_{C}$ is not small. Since this additional stabilizing force is also proportional to $U^{2}$, higher values of the lift coefficient $f$ are required to compensate for it, leading to a higher critical lift parameter $f$, as found in figure $8(b)$.

Using a similar argument, we may predict an additional stabilizing (respectively, destabilizing) force proportional to $\left(c_{N} / c_{T}-1\right) U^{2}$ when $c_{N} / c_{T}$ is lower (respectively, higher) than 1; this can be seen in (2.11) or (2.14). This also results in a higher critical lift coefficient, consistent with the results of Semler et al. (2002) for short cylinders and our results for long cylinders.

\section{Concluding remarks}

The possibility that very long cylinders with a free downstream end may flutter when subjected to axial flow has always been a concern in the design of long towed underwater arrays or floating containers. Because of the difficulty in realizing laboratory experiments with such slender systems, analytical models have been developed that involved approximations regarding the respective roles of axial tension and flexural stiffness in the stability of the cylinder. These models led to the conclusion that, though divergence was possible, the risk of flutter would disappear if the cylinder were long enough. Simultaneously, extensive numerical and experimental analyses of the linear and nonlinear stability of shorter cylinders were conducted, which showed the adequacy of the models for the fluid forces. These analyses made use of dimensionless parameters based on the cylinder length, which could be misleading in determining the effect of length on stability, and also in deciding whether long cylinders would flutter.

By introducing an alternative dimensionless scheme for the same equations and by exploring a larger range of values of the cylinder length, we found that flutter can also exist for very long cylinders, provided that the downstream end is sufficiently well streamlined. This flutter regime, which could not be found in previous analytical solutions because of the simplifying assumptions made therein, is, in fact, very similar to that of shorter cylinders, both in terms of linear and nonlinear dynamics. Indeed, this similarity is so strong that a very long cylinder can, to some extent, be modelled as a short cylinder of length $L_{C}$, which is the length where friction-related flow-induced tension is negligible compared to the bending stiffness. This length is also the scale where most of the deformation occurs. The main specificity of long cylinders is that the friction-induced tension stiffens the upstream part, so that a higher lift coefficient is required at the downstream end for flutter to arise. 
The spatially restricted form of instabilities found here for long cylinders is very similar to that observed recently in experiments and models of hanging fluid-conveying pipes, hanging ribbons with axial flow or inclined filaments in flow. They all belong to a type of edge flutter, where the flutter wavelength is not fixed by the scale of the structure but by the boundary-layer thickness of the structural displacement defined by a balance between local bending stiffness and fluid forces.

The support of the DGA grant 036000063 for the sabbatical stay of E. L. at McGill University is gratefully acknowleged, as is the support by NSERC of Canada to M.P.P. and Y.M.-S.

\section{REFERENCES}

Bhattacharyya, S. K., Vendhan, C. P. \& Sudarsan, K. 2000 The finite element method for hydroelastic instability of underwater towed cylindrical structures. J. Sound Vib. 237, 119-143.

Doaré, O. \& DE LANGRE, E. 2002 The flow-induced instability of long hanging pipes. Eur. J. Mech. A/Solids 21, 857-867.

Doedel, E. \& Kernéves, J. 1986 AUTO: Software for continuation problems in ordinary differential equations with applications. Tech. Rep. California Institute of Technology. Applied Mathematics.

Done, G. T. S. \& Simpson, A. 1977 Dynamic stabilty of certain conservative and non-conservative systems. IMechE J. Engng Sci. 19, 251-263.

Dowling, A. P. 1988 The dynamics of towed flexible cylinders. Part 1. Neutrally buoyant elements. J. Fluid Mech. 187, 507-532.

de Langre, E., Doaré, O. \& Pellet, F. 2001 Follower force on a semi-infinite beam loaded by its own weight: model and experiments. C. R. Acad. Sci. Paris II B 329, 175-178.

LeE, T. S. 1981 Stability analysis of the Ortloff-Ives equation. J. Fluid Mech. 110, 293-295.

Lemaitre, C., Hémon, P. \& De Langre, E. 2005 Instability of a long ribbon hanging in axial air flow. J. Fluids Struct. 21, 913-925.

Lighthill, M. J. 1960 Note on the swimming of slender fish. J. Fluid Mech. 9, 305-317.

Lopes, J. L., PAïDoussis, M. P. \& SemLer, C. 2002 Linear and nonlinear dynamics of cantilevered cylinders in axial flow. Part 2. The equations of motion. J. Fluids Struct. 16, 715-737.

Ni, C. C. \& Hansen, R. J. 1978 An experimental study of the flow-induced motions of a flexible cylinder in axial flow. Trans. ASME I: J. Fluids Engng 100, 389-394.

Ortloff, C. R. \& Ives, J. 1969 On the dynamic motion of a thin flexible cylinder in a viscous stream. J. Fluid Mech. 38, 713-720.

PAïDoussis, M. P. 1966 Dynamics of flexible slender cylinders in axial flow. Part 2. Experiments. J. Fluid Mech. 26, 737-751.

PAïDoussis, M. P. 1973 Dynamics of cylindrical structures in axial flow. J. Sound Vib. 29, 365-385.

PAïDoussis, M. P. 1998 Fluid-Structure Interactions: Slender Structures and Axial Flows, vol. 1. Academic.

PAïDoussis, M. P. 2003 Fluid-Structure Interactions: Slender Structures and Axial Flows, vol. 2. Elsevier.

Païdoussis, M. P., Grinevich, E., Adamovic, D. \& Semler, C. 2002 Linear and nonlinear dynamics of cantilevered cylinders in axial flow. Part 1. Physical dynamics. J. Fluids Struct. 16, 691-713.

PAO, H. P. 1970 Dynamics stability of a towed thin flexible cylinder. AIAA J. Hydronaut. 4, 144-150.

Schouveiler, L., Eloy, C. \& Le Gal, P. 2005 Flow-induced vibrations of high mass ratio flexible filaments freely hanging in a flow. Phys. Fluids 17, 047104.

Semler, C., Lopes, J. L., Augu, N. \& Païdoussis, M. P. 2002 Linear and nonlinear dynamics of cantilevered cylinders in axial flow. Part 3. Nonlinear dynamics. J. Fluids Struct. 16, 739-759.

Sudarsan, K., Bhattacharyya, S. K. \& Vendhan, C. P. 1997 An experimental study of hydroelastic instability of flexible towed underwater cylindrical structures. In Proc. 16th OMAE Conf., Yokohama, Japan, pp. 73-80. ASME.

SugiYama, Y. \& KawaGoE, H. 1975 Vibration and stability of elastic columns under the combined action of uniformly distributed vertical and tangential forces. J. Sound Vib. 38, 341-355. 
Triantafyllou, G. S. \& Chryssostomidis, C. 1984 Analytical determination of the buckling speed of towed slender cylindrical beams. Trans. ASME J. Energy Resources Technol. 106, 247-249.

Triantafyllou, G. S. \& Chryssostomidis, C. 1985 Stability of a string in axial flow. Trans. ASME J. Energy Resources Technol. 107, 421-425.

Zhang, J., Childress, S., Libchaber, A. \& Shelley, M. 2000 Flexible filaments in a flowing soap film as a model for one-dimensional flags in a two-dimensional wind. Nature 408, 835-839. 